\title{
Efficient hierarchical method for background subtraction
}

\author{
Yu-Ting Chen ${ }^{\mathrm{a}, \mathrm{b}}$, Chu-Song Chen ${ }^{\mathrm{a}, \mathrm{c}, *}$, Chun-Rong Huang ${ }^{\mathrm{a}}$, Yi-Ping Hung ${ }^{\mathrm{a}, \mathrm{b}, \mathrm{c}}$ \\ anstitute of Information Science, Academia Sinica, 128 Academia Road, Section 2, Nankang, Taipei 115, Taiwan \\ ${ }^{\mathrm{b}}$ Department of Computer Science and Information Engineering, National Taiwan University, 1 Roosevelt Road, Section 4, Taipei 106, Taiwan \\ ${ }^{\mathrm{c}}$ Graduate Institute of Networking and Multimedia, National Taiwan University, 1 Roosevelt Road, Section 4, Taipei 106, Taiwan
}

Received 5 June 2006; received in revised form 14 November 2006; accepted 21 November 2006

\begin{abstract}
Detecting moving objects by using an adaptive background model is a critical component for many vision-based applications. Most background models were maintained in pixel-based forms, while some approaches began to study block-based representations which are more robust to non-stationary backgrounds. In this paper, we propose a method that combines pixel-based and block-based approaches into a single framework. We show that efficient hierarchical backgrounds can be built by considering that these two approaches are complementary to each other. In addition, a novel descriptor is proposed for block-based background modeling in the coarse level of the hierarchy. Quantitative evaluations show that the proposed hierarchical method can provide better results than existing single-level approaches.
\end{abstract}

(C) 2006 Pattern Recognition Society. Published by Elsevier Ltd. All rights reserved.

Keywords: Hierarchical background modeling; Background subtraction; Contrast histogram; Non-stationary background; Object detection; Video surveillance

\section{Introduction}

Background modeling is an important module for many vision-based applications, such as visual surveillance and human gesture analysis. To detect moving objects, each incoming frame is compared with the background model learned from previous frames to classify the scene into foreground and background. A difficulty encountered in background modeling is that backgrounds are usually non-stationary in practice. Nonstationary backgrounds would be caused by waving leaves, fluttering flags, ripple water, fluorescent light, monitor flicker, and so on. Even when the background is static, camera jittering and signal noise may still cause non-stationary problems. Furthermore, shadows [1-3] and sudden lighting changes [4] are also important issues. In Ref. [5], Toyama et al. summarized 10 important problems in background subtraction. Except for these problems, real-time performance is also an important problem.

\footnotetext{
* Corresponding author. Institute of Information Science, Academia Sinica, 128 Academia Road, Section 2, Nankang, Taipei 115, Taiwan.

Tel.: +886227883799x1310 ; fax: +886227824814 .

E-mail address: song@iis.sinica.edu.tw (C.-S. Chen).
}

Most background modeling methods are pixel-based. Gaussian distribution has become a popular choice for modeling. Since the background is often non-stationary, a single Gaussian model used in Refs. [2,6] is not sufficient for its representation. In Ref. [7], Stauffer and Grimson proposed Mixture of Gaussians (MoG) by using $k$ Gaussians to model each pixel. In MoG, an online $K$-means approximation was used instead of using the exact Expectation-Maximization (EM) algorithm. The MoG approach is modified or extended in several researches. For example, instead of using RGB color as features, Harville et al. [8] used YUV color plus depth measured by a stereo camera as features instead of using RGB color. Harville [9] introduced a framework to guide pixel-level evolution in Ref. [8] with high-level information. In Ref. [10], Lee proposed an effective learning algorithm for MoG.

Instead of Gaussian mixtures, Ridder et al. [11] used Kalman filter for adaptive background estimation. In Ref. [12], Zhong and Sclaroff developed a foreground-background segmentation algorithm via a robust Kalman filter to segment the foreground objects from time-varying and textured backgrounds. Stenger et al. [13] presented a framework for Hidden-Markov-Model topology and parameter estimation in an online and dynamic fashion. In Ref. [5], Toyama et al. proposed Wallflower to use 
pixel-level, region-level, and frame-level components to automatically identify people, objects, or events of interest in different kinds of environments. Elgammal et al. [1] proposed a non-parametric background subtraction method utilizing Parzen-window density estimation for representing the background. In Ref. [14], Kim et al. presented a real-time algorithm called Codebook that is efficient in either memory or speed.

Pixel-based methods model each pixel independently. Approaches of this type have the advantage of extracting detailed shapes of moving objects, but may suffer from the drawback that their segmentation results are sensitive to non-stationary scenes or backgrounds. Though some approaches such as Refs. [1,4] considered relative relationships between neighboring pixels in either space or time domain, a local vibration of the scene may still cause problems of false detection. In fact, high false-alarm rates have become a serious problem for many practical visual-surveillance systems.

Recently, some researches used block-based approaches instead of pixel-based approaches for background modeling and subtraction. In block-based approach, an image is often divided into overlapped or non-overlapped blocks, and specific block features are used for background modeling. Since a block can monitor more global changes in the scene than a single pixel, block-based approaches are insensitive to local movements and are more capable of dealing with non-stationary backgrounds. In addition, by using efficient features for each block of images, block-based approach is possible to be implemented very fast. Nevertheless, a primary limitation of block-based approach is that only a coarse-resolution foreground can be extracted, and so it is not suitable for applications requiring detailed shape information.

In recent years, researches for block-based background modeling are proposed. In Ref. [15], Matsuyama et al. proposed normalized vector distance (NVD) to measure the correlations between blocks. In Ref. [16], Mason et al. calculated edge and color histograms in each block as features to describe the block, and histogram similarity is computed to detect the foreground region. In Ref. [17], Monnet et al. used incremental PCA and an online auto-regressive model to predict a dynamic scene. In Ref. [18], Heikkilä et al. used local binary pattern (LBP) [19] histogram to capture background statistics of each block.

Both pixel- and block-based approaches have their pros and cons. An interesting issue is that they are complementary to each other. The simplest way to combine them might be running these two approaches independently, and then taking the intersections or unions of the detected foreground regions as the results. However, this combination is not the most efficient way. In this paper, a hierarchical method is proposed to combine a block-based approach, referred to as a coarse-level approach, and a pixel-based approach, referred to as a fine-level approach, in an asymmetric feed-forward framework. A novel discriminative descriptor called contrast histogram that is extended from Ref. [20] is used as a feature to describe each block, and Gaussian mixtures are used for maintaining a coarse-level model. For the fine-level model, existing pixel-based methods can be adopted with merely a slight modification, and a feed-forward framework is introduced to effectively dispatch the detected coarse-level information to the fine-level stage.

The paper is organized as follows: In Section 2, coarse-level background modeling using contrast histogram is introduced. The strategy for combining coarse- and fine-level models is presented in Section 3. Experimental results are shown in Section 4. Conclusion and future work are given in Section 5.

\section{Coarse-level modeling}

In coarse level, an efficient descriptor is expected to be built for an image block. In object recognition, invariant descriptors such as scale-invariant feature transformation (SIFT) [21] have shown their convincing performance for representing a region centered at a feature point. In SIFT, significant keypoints are identified and each keypoint is assigned with a descriptor composed of the orientation histograms computed from the gradient magnitudes and orientations sampled around the keypoint. Though SIFT has shown its powerfulness in several applications, it is not suitable for describing a background region in our experience. The primary difficulty is that the SIFT descriptor is suitable for representing clutter scenes. However, when some backgrounds are featureless, the gradient information used in SIFT causes unstable representations.

In Ref. [19], LBP has shown its powerful means for texture recognition. To represent a feature point, the circular neighboring pixels are labeled by thresholding the difference between neighboring pixels and the center (feature) pixel. Then the labeled results are considered as a binary number (LBP code). In Ref. [18], Heikkilä et al. used histogram of LBP codes in image blocks to capture background statistics. Nevertheless, LBP histogram is not suitable to describe non-stationary backgrounds according to our experimental results (see Section 4).

In this research, we construct the contrast histogram descriptor extended from Ref. [20] to describe each block directly based on the pixel colors in a block. The proposed contrast histogram is insensitive to center-point drifts and pixel reshuffles.

\subsection{Contrast histogram of gray-level images}

Given an image $I$, we first smooth this image by applying Gaussian kernels and obtain a Gaussian smoothed image $L$ :

$L(\mathbf{p}, \sigma)=G(\mathbf{p}, \sigma) * I(\mathbf{p})$,

where $\mathbf{p}$ is a pixel at location $(x, y), *$ is convolution, and $G(\mathbf{p}, \sigma)$ is a Gaussian function with variance $\sigma^{2}$.

After dividing an image into blocks, our next step is to build a descriptor for each block $\mathbf{B}_{c}$. One obvious approach would be to sample the local image intensities in $\mathbf{B}_{c}$ as a template and perform template matching by using normalized correlation as in Ref. [15]. However, this method is sensitive to noise [5].

Our approach does not involve the gradient computation, and is stable to compute. The descriptor is constructed based on the contrast value defined below:

$C\left(\mathbf{p}, \mathbf{p}_{c}\right)=L(\mathbf{p})-L\left(\mathbf{p}_{c}\right)$, 
a

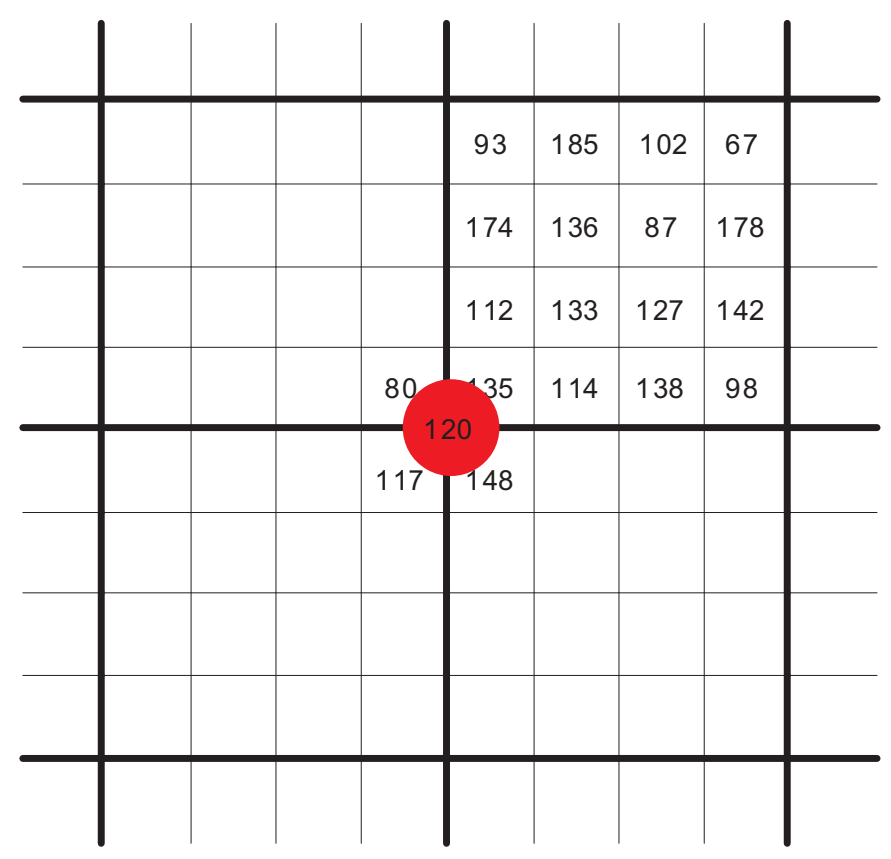

b

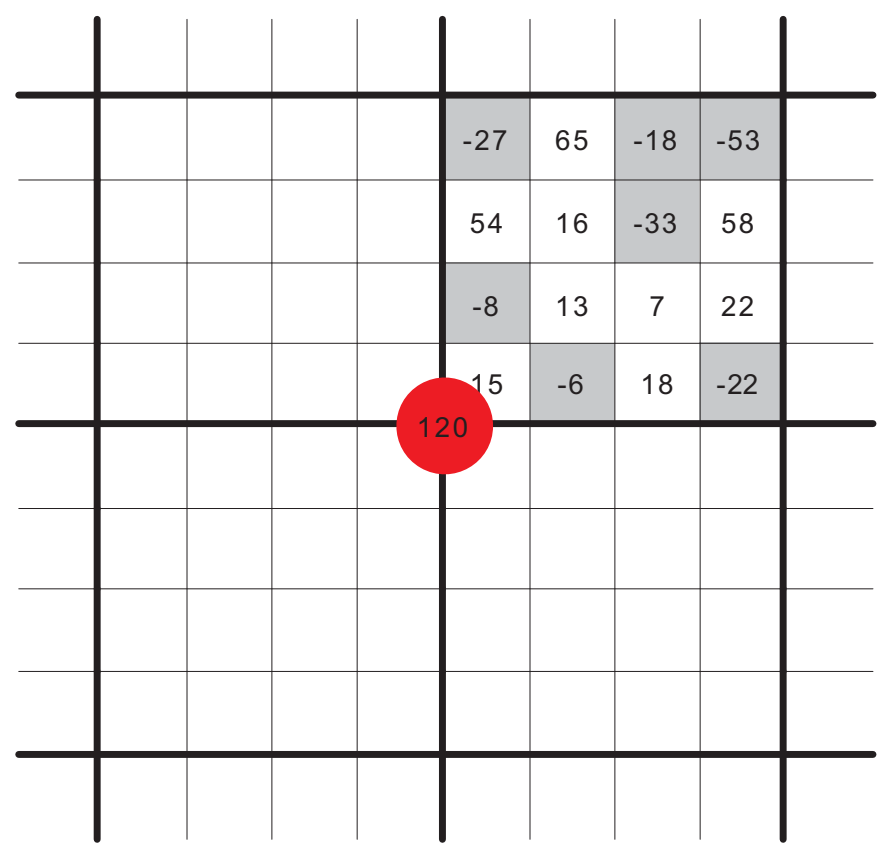

Fig. 1. An example of contrast histogram. Assume that the block size is $8 \times 8$ and the red point is the averaged center point $\mathbf{p}_{c}$. Each block is separated into four quadrants. (a) Original gray-scale image. The averaged value of the center point $\mathbf{p}_{c}$ is $120(=(80+135+117+148) / 4)$. (b) The contrast values of the pixels in the first quadrant are calculated, and the pixels with positive and negative contrast values are shown in white and gray cells, respectively. The values of the positive contrast histogram bin, $\mathrm{CH}_{q_{0}}^{+}\left(\mathbf{p}_{c}\right)$, and negative contrast histogram bin, $\mathrm{CH}_{q_{0}}^{-}\left(\mathbf{p}_{c}\right)$, are $29.79(=(65+54+16+58+13+7+22+15+18) / 9)$ and $-23.86(=(-27-18-53-33-8-6-22) / 7)$, respectively.

where $\mathbf{p}$ is a pixel in block $\mathbf{B}_{c}$. Since the center pixel $\mathbf{p}_{c}$ in block $\mathbf{B}_{c}$ does not necessarily exist, the value of $\mathbf{p}_{c}$ is estimated by averaging the four center pixels of $\mathbf{B}_{c}$. Employing the contrast values implies the insensitiveness to lighting changes. It is computationally efficient because only pixel-based subtraction is required.

Similar to SIFT, we separate each block into four quadrant bins, and then the contrast values in each quadrant bin are used to build the descriptor. Directly using the contrast values of all the pixels in a block as a descriptor is much too detailed since the shapes and their sampled representation may vary from one instance to another. In our approach, we employ the contrast distribution in each quadrant bin to build a robust descriptor. One obvious approach would be to sum up the contrast values in each quadrant bin. It is not a good idea because the summations of positive and negative contrast values may decrease the responses of bins. In our framework, we compute positive and negative contrast-value histograms for each quadrant bin $q_{i}$. The positive contrast histogram $\mathrm{CH}_{q_{i}}^{+}\left(\mathbf{p}_{c}\right)$ of $q_{i}$ with respect to $\mathbf{p}_{c}$ is defined as follows:

$C H_{q_{i}}^{+}\left(\mathbf{p}_{c}\right)=\frac{\sum\left\{C\left(\mathbf{p}, \mathbf{p}_{c}\right) \mid \mathbf{p} \in q_{i} \text { and } C\left(\mathbf{p}, \mathbf{p}_{c}\right)>0\right\}}{\#_{q_{i}}^{+}}$,

where $i=0, \ldots, 3$ and $\#_{q_{i}}^{+}$is the number of pixels with positive contrast values in $q_{i}$. Similarly, the negative contrast histogram
$\mathrm{CH}_{q_{i}}^{-}\left(\mathbf{p}_{c}\right)$ is defined as

$C H_{q_{i}}^{-}\left(\mathbf{p}_{c}\right)=\frac{\sum\left\{C\left(\mathbf{p}, \mathbf{p}_{c}\right) \mid \mathbf{p} \in q_{i} \text { and } C\left(\mathbf{p}, \mathbf{p}_{c}\right)<0\right\}}{\#_{q_{i}}^{-}}$,

where $\#_{q_{i}}^{-}$is the number of pixels with negative contrast values in $q_{i}$. The contrast-histogram descriptor of a gray-level block $\mathbf{B}_{c}$ is an eight-dimensional vector defined as

$$
\begin{aligned}
\mathrm{CH}_{G}\left(\mathbf{p}_{c}\right)= & \left(\mathrm{CH}_{q_{0}}^{+}, \mathrm{CH}_{q_{0}}^{-}, \mathrm{CH}_{q_{1}}^{+}, \mathrm{CH}_{q_{1}}^{-}, \mathrm{CH}_{q_{2}}^{+},\right. \\
& \left.\mathrm{CH}_{q_{2}}^{-}, \mathrm{CH}_{q_{3}}^{+}, \mathrm{CH}_{q_{3}}^{-}\right),
\end{aligned}
$$

where $\mathrm{CH}_{G}\left(\mathbf{p}_{c}\right) \in R^{8}$. An example is illustrated in Fig. 1.

\subsection{Contrast histogram of color images}

The descriptor introduced above is for gray-level images. It can be realized very fast and can effectively extract foreground regions for many situations. However, gray-value information is not sufficient for identifying the foreground objects in some cases. For example, different colors may have similar gray values and are indistinguishable. Thus, we extend the descriptor to a color version.

Unlike the common extension of duplicating the gray-value method for each of the three color channels, we allow the use of cross-color contrast information. Let $j \in\{R, G, B\}$ and $k \in\{R, G, B\}$ stand for the color channels of $\mathbf{p}$ and $\mathbf{p}_{c}$, 
respectively. The positive and negative color contrast histograms are defined as

$$
\begin{aligned}
& C H_{q_{i}}^{j, k ;+}\left(\mathbf{p}_{c}\right) \\
& \quad=\frac{\sum\left\{C^{j, k}\left(\mathbf{p}, \mathbf{p}_{c}\right) \mid \mathbf{p} \in q_{i} \text { and } C^{j, k}\left(\mathbf{p}, \mathbf{p}_{c}\right)>0\right\}}{\#_{q_{i}}^{+}}
\end{aligned}
$$

and

$$
\begin{aligned}
& C H_{q_{i}}^{j, k ;-}\left(\mathbf{p}_{c}\right) \\
& =\frac{\sum\left\{C^{j, k}\left(\mathbf{p}, \mathbf{p}_{c}\right) \mid \mathbf{p} \in q_{i} \text { and } C^{j, k}\left(\mathbf{p}, \mathbf{p}_{c}\right)<0\right\}}{\#_{q_{i}}^{-}},
\end{aligned}
$$

respectively, where $C^{j, k}\left(\mathbf{p}, \mathbf{p}_{c}\right)$ is the contrast value between the $j$ channel of $\mathbf{p}$ and the $k$ channel of $\mathbf{p}_{c}$.

There are nine combinations of the pair $(j, k)$, which introduces a $72(=9 \times 4 \times 2)$-dimensional description vector for each block. However, it is not necessary to use all the pairs. Instead, we only pick up the six pairs, $(j, k) \in$ $\{(R, R),(R, G),(R, B),(G, G),(G, B),(B, B)\}$ and thus a $48(=6 \times 4 \times 2)$-dimensional color descriptor, $\mathrm{CH}_{C}\left(\mathbf{p}_{c}\right) \in R^{48}$, is employed for efficient construction and matching.

\subsection{Background modeling by contrast histograms}

In coarse-level background modeling, $\mathrm{CH}_{G}\left(\mathbf{p}_{c}\right)$ or $\mathrm{CH}_{C}\left(\mathbf{p}_{c}\right)$ is used as the feature of each block. The previous contrasthistogram history of each block, $\left\{\mathbf{x}_{1}, \mathbf{x}_{2}, \ldots, \mathbf{x}_{t}\right\}$, is modeled by $K$ weighted Gaussian distributions. The weight of the $k$ th Gaussian at time $t$ is denoted as $\omega_{k, t}$ and $K$ weights are normalized such that $\sum_{k=1}^{K} \omega_{k, t}=1$. The probability of the event that the currently observed feature $\mathbf{x}_{t+1}$ is a background block is

$P\left(\mathbf{x}_{t+1}\right)=\sum_{k=1}^{K} \omega_{k, t} \times \eta\left(\mathbf{x}_{t+1}, \mu_{k, t}, \Sigma_{k, t}\right)$,

where $\mu_{k, t}$ and $\Sigma_{k, t}$ are the mean vector and covariance matrix of the $k$ th Gaussian at time $t, \eta$ is a Gaussian probability density function, and the covariance matrix is assumed to be the form:

$\Sigma_{k, t}=\sigma_{k, t}^{2} I$.

To realize coarse-level model, an online $K$-means algorithm presented in Ref. [7] is applied to recent contrast-histogram history of each block. In our experiments, $K$ is set as 3. Every new observed feature vector, $\mathbf{x}_{t+1}$, is checked against the existing $K$ Gaussians, until a match is found. The "match" is defined as the situation that $\mathbf{x}_{t+1}$ lies within $m$ standard deviations of a Gaussian. In our case, $m$ is set between 2.5 and 3. If none of the $K$ Gaussians matches the current vector $\mathbf{x}_{t+1}$, the least probable Gaussian is replaced with a new Gaussian with $\mathbf{x}_{t+1}$ as its mean value, an initial high variance, and an initial low weight. If one of the $K$ Gaussians is matched, the matched Gaussian is updated as follows:

$\mu_{t+1}=(1-\rho) \mu_{t}+\rho \mathbf{x}_{t+1}$,

$\sigma_{t+1}^{2}=(1-\rho) \sigma_{t}^{2}+\rho\left(\mathbf{x}_{t+1}-\mu_{t+1}\right)^{\mathrm{T}}\left(\mathbf{x}_{t+1}-\mu_{t+1}\right)$,

where $\rho=\alpha \eta\left(\mathbf{x}_{t+1} \mid \mu_{t}, \sigma_{t}\right)$ and $\alpha$ is a user-defined learning rate. The weights of $K$ Gaussians are adjusted as follows:

$\omega_{t+1}=(1-\alpha) \omega_{t}+\alpha M_{t+1}$,

where $M_{t+1}=1$ is set for the matched Gaussian and $M_{t+1}=0$ is set for the remaining Gaussians. Furthermore, weights of $K$ Gaussians are normalized to ensure $\sum_{k=1}^{K} \omega_{k, t}=1$.

Since the Gaussian with a high weight and a low variance has more supporting evidence as a background, $K$ weighted Gaussians are ordered by the value of $\omega / \sigma$. Then the first $B$ Gaussians are chosen as the background models, where

$B=\arg \min _{b}\left(\sum_{k=1}^{b} \omega_{k}>T\right)$,

where $T$ is a measure of the minimum portion of the data for which should be accounted by the background. If observed feature vector $\mathbf{x}_{t+1}$ matches these first $B$ Gaussians, the corresponding block is classified as background; otherwise, the block is foreground.

\subsection{Coarse-level experimental results}

Some experiments were performed to verify the effectiveness of the proposed coarse-level methods. Figs. 2 and 3 show the results of a non-stationary outdoor scene (with fountains and waving leaves) and a static indoor scene, respectively. The frame resolution of the processed videos is $320 \times 240$ and the block size is $8 \times 8$ for all the experiments. Both the gray-level and color contrast histograms, $\mathrm{CH}_{G}\left(\mathbf{p}_{c}\right)$ and $C H_{C}\left(\mathbf{p}_{c}\right)$, are used
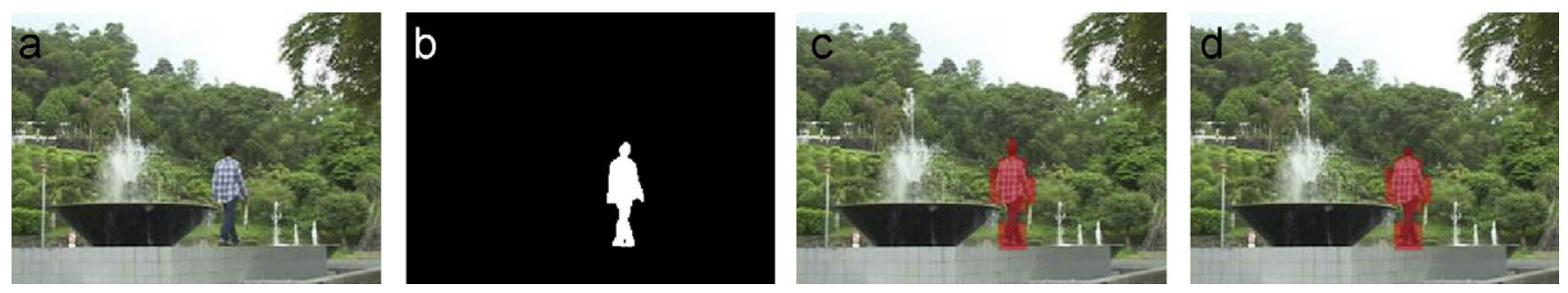

Fig. 2. The coarse-level results of frame $\# 500$ of an outdoor video (with fountains and waving leaves): (a) original source image; (b) ground truth image. The red masks in (c) and (d) are the results of gray-level and color contrast histograms, respectively. 

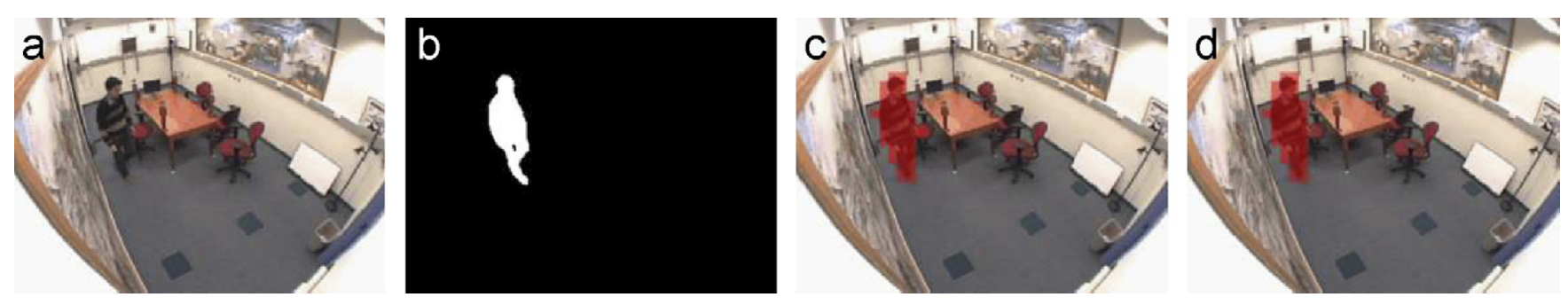

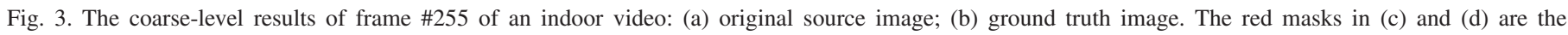
results of gray-level and color contrast histograms, respectively.

for background extraction, where the former results are shown in Figs. 2(c) and 3(c) and the latter results are shown in Figs. 2(d) and 3(d), respectively. The results reveal that both the gray-level and color contrast histograms are effective for background modeling of either outdoor or indoor scenes, although the result of the color contrast histogram is a bit more accurate. Yet note that the gray-level contrast histogram is more efficient to compute. The processing speed of the graylevel version for the outdoor and indoor experiments are 163 and $136 \mathrm{fps}$ (frames per second), respectively, while those of the color version are 67 and $66 \mathrm{fps}$, respectively, by using a PC with a $3.4 \mathrm{GHz}$ processor and $768 \mathrm{MB}$ memory. The high computation efficiency makes our method suitable for real-time visual-surveillance applications requiring multiple video sources to be processed simultaneously in a single machine, and also provides a good start to initiate a fine-level result. Some further experiments and comparison with other block-based methods will be given in Section 4 .

\section{Hierarchical background models}

Though the coarse-level outputs are sufficient for many applications such as intruder detections, some applications need more detailed foregrounds to be segmented.

An essential advantage of incorporating a coarse-level approach into a pixel-level approach is that non-stationary scenes can be processed well since more global information has been used. An issue remained to be discussed is the combination efficiency. A straightforward combination is to run the coarseand fine-level methods independently and then join their foreground intersection regions. Beyond this scope, we hope that the integrated computation time is shorter than the sum of those of running the coarse- and fine-level methods.

By incorporating the pixel-based segmentation into the coarse-level results, a two-stage hierarchical approach is introduced. In the following, we give a general description of pixel-based methods, and then introduce the combination framework.

\subsection{A general description of pixel-based background modeling}

Most of the existing pixel-based background-modeling methods share a similar framework. We give a general description of the common framework in the following.

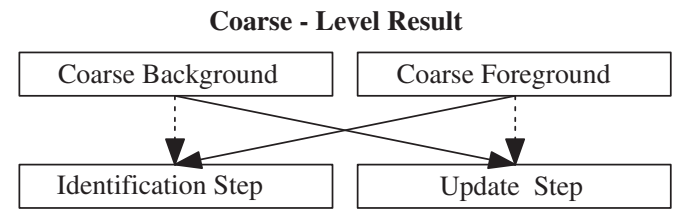

Fine - Level Result

Fig. 4. Asymmetric feed-forward strategy for coarse background and foreground regions. Solid and dashed lines mean the executed and neglected step in fine level, respectively.

A pixel-based approach can be generally characterized as a quadruple $\left\{F, M_{(t)}, \boldsymbol{\Phi}, \boldsymbol{\Gamma}\right\}$. The first element $F$ depicts the feature extracted for a pixel, which might be gray/color values $[1,7,9,14]$, depth [8,9], gradient intensities [4], or co-occurred color pairs [4].

The second element $M_{(t)}$ consists of the background models recorded at time $t$ for the pixel. For example, in MoG approach [7], each model is represented as a single Gaussian distribution in the mixture. In the Codebook approach [14] the model is characterized by a cylindrical region in the color space. Note that almost all the methods maintained $M_{(t)}=\left\{M_{(t)}^{S}, M_{(t)}^{P}\right\}$, where $M_{(t)}^{S}$ and $M_{(t)}^{P}$ are the sets of stable and potential background models, respectively. For example, in MoG [7], the first $B$ Gaussian densities constitute $M_{(t)}^{S}$ and the others constitute $M_{(t)}^{P}$.

The third element $\boldsymbol{\Phi}$ is a function determining whether a given pixel $q$ at time $t$ is background based on the pixel feature $F(q)$ and the stable background model $M_{(t)}^{S}$ :

$\{1,0\} \leftarrow \boldsymbol{\Phi}\left[F(q), M_{(t)}^{S}\right]$,

where $F(q)$ is the feature of $q$, and 1 and 0 stand for background and foreground, respectively. Note that only the stable model $M_{(t)}^{S}$ is involved in the determination. The fourth element $\boldsymbol{\Gamma}$ is another function that updates the model and generates a new model at time $t+1$ based on the pixel feature $F(q)$ and the current model $M_{(t)}$ :

$M_{(t+1)} \leftarrow \Gamma\left[F(q), M_{(t)}\right]$,

and a new pair of models, $M_{(t+1)}=\left\{M_{(t+1)}^{S}, M_{(t+1)}^{P}\right\}$, is obtained. To realize $\Gamma$ typically involves the search of the most fit model to $F(q)$ in $M_{(t)}$. 
Table 1

Five benchmark sequences used in the experiments

\begin{tabular}{|c|c|c|c|c|c|}
\hline Sequence name & Fountains & Waving leaves & Ocean waves & Waving river & Moving escalator \\
\hline \multicolumn{6}{|l|}{ Sequence image } \\
\hline Frame number & 185 & 420 & 640 & 230 & 60 \\
\hline Sequence type & Dynamic & Dynamic & Dynamic & Texture & Texture \\
\hline Sequence length & 950 & 1016 & 903 & 291 & 172 \\
\hline Frames for training & 100 & 100 & 450 & 170 & 50 \\
\hline
\end{tabular}

\subsection{Asymmetric feed-forwarding}

Our combination is based on the scenario that the coarsely detected foreground is further refined in fine level. It would be straightforward if only foreground region refinements are involved. However, since the fine-level background model needs to be updated to maintain its accuracy, the fine-level background updating shall also be involved in the combination. We conduct an integration strategy as below.

- Fine processing of coarse background: For those pixels in the coarse background, fine-level model updating is performed but fine-level background identification (or equivalently, foreground detection) is ignored, i.e., the function $\boldsymbol{\Gamma}$ is implemented but $\boldsymbol{\Phi}$ is not.

- Fine processing of coarse foreground: For those pixels in the coarse foreground, fine-level background identification is performed but model updating is ignored, i.e., the function $\boldsymbol{\Phi}$ is implemented but $\boldsymbol{\Gamma}$ is not.

The above processes yield an asymmetric feed-forward strategy from coarse to fine levels, as illustrated in Fig. 4. Note that if we do not neglect the dashed-line steps in Fig. 4, it is equivalent to running the coarse and fine methods independently and then intersect their foregrounds obtained.

The reason of adopting this strategy is given below. First, since the coarse-level method is based on blocks instead of pixels, being justified as background in this level is convincing since a group of pixels (contained in a block) are used for the justification. Thus, we can neglect the pixel-based identification step in this case without sacrificing the segmentation performance. However, we still need to update the fine-level model for each pixel or we may lose the incremental-adaptation information of the fine-level model. When a block is identified as foreground in the coarse level, the updating step is neglected in our approach since the detected coarse foreground may contain true foregrounds or unstable backgrounds, such as shadows and signal noise.
By applying this strategy, the total computation time can be reduced. It is because only the function $\boldsymbol{\Phi}$ is required to be realized in the fine-identification step. Compared to the way of performing the coarse and fine steps independently and then intersecting the results, we have only to search the model in $M_{(t)}^{S}$ instead of the whole $M_{(t)}$ for model fitting, and thus save the time of searching $M_{(t)}^{P}$ in fine processing of the coarse foregrounds.

\section{Realization and experimental results}

\subsection{Implementation}

Our approach does not restrict the pixel-based approaches being used for fine level. In our implementation, a stateof-the-art pixel-based method, MoG [7], is adopted for the fine-level step, and the color version of the contrast-histogram approach is adopted for coarse level. To reduce false positives (background pixels misclassified as foreground pixels), post-processing steps are performed on both coarse- and finelevel results. In coarse level, foreground blocks are grouped into connected components (according to the 4-adjacency definition [22]), and the foreground components with no more than three blocks are considered as false positive regions and are removed. Similarly, in fine level, connected components are also found based on the foreground pixels, and the foreground components with no more than five pixels are removed.

\subsection{Experimental results}

To evaluate the proposed background subtraction methods, three dynamic outdoor sequences and two sequences with dynamic textured backgrounds used in Ref. [12] are adopted as the benchmarks as summarized in Table 1. The three dynamic outdoor sequences include the fountains sequence with four fountains and waving leaves in upper-right, the waving leaves sequence with large-area waving leaves, and the ocean waves 


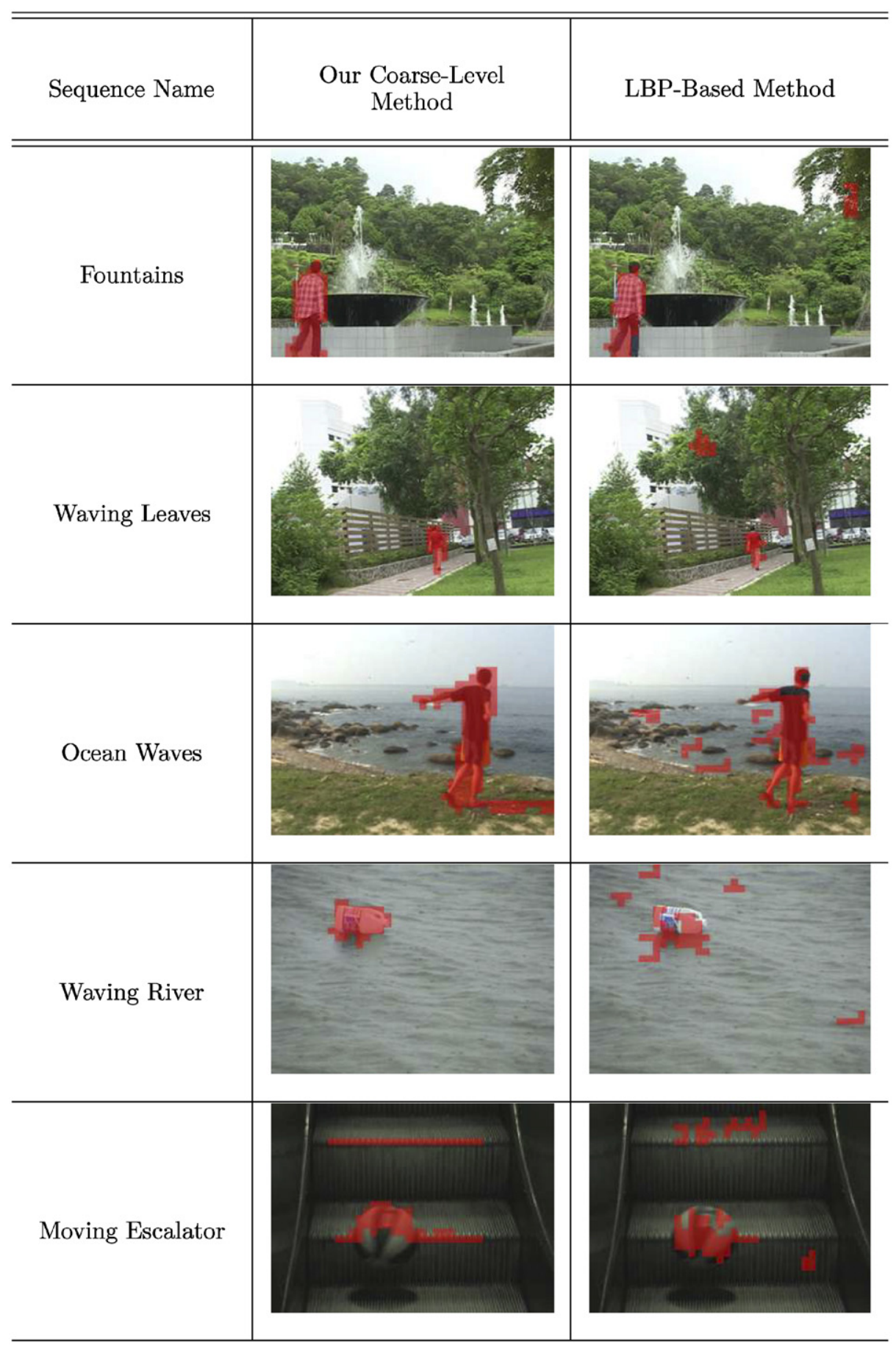

Fig. 5. Coarse-level results of five benchmark sequences shown in Table 1. LBP-based textured method was implemented for comparison.

sequence with ocean waves hitting the rocky coast. The two dynamic textured sequences include waving river sequence with ripples on the surface of the river and moving escalator sequence with regular time-varying movement of escalator. The image resolutions are $320 \times 240$ for all of the benchmark sequences.

Fig. 5 shows our coarse-level results with at most 3 weighted Gaussian mixtures being used for each block and the results of LBP-based textured method [18] are also shown for comparison. Note that both methods are combined with a post processing of removing connected components with no more than three blocks. The average processing speeds of these two methods are shown in Table 2. From Fig. 5 and Table 2, our coarse-level algorithm can provide more promising results and higher computation speeds than LBP-based textured method.

Fig. 6 shows the comparison results of the proposed hierarchical method by applying MoG as the fine-level method and 


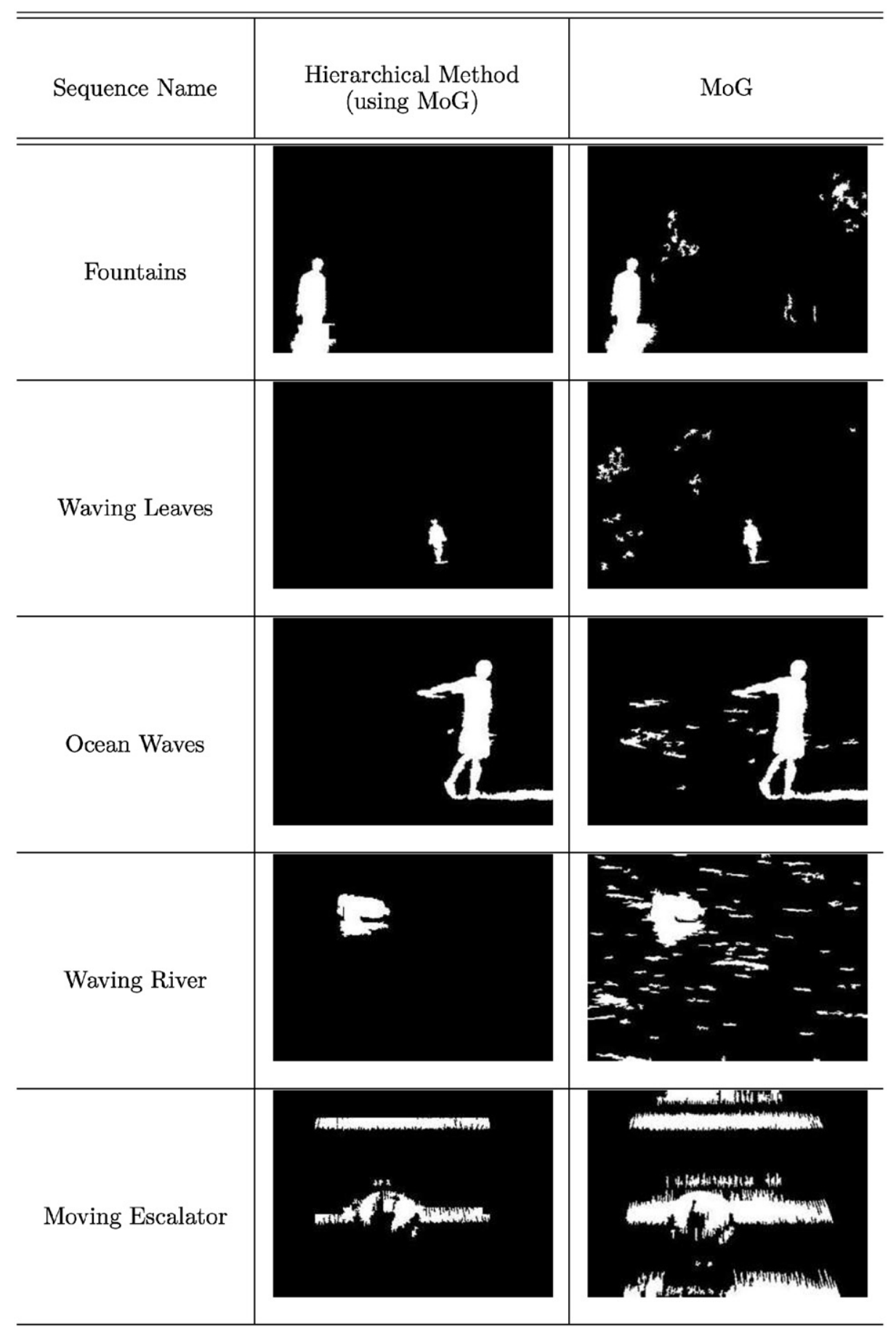

Fig. 6. Results of the proposed hierarchical approach with MoG being its fine-level method and the original MoG method.

original MoG method, where both our fine-level method and MoG are followed by a post processing of removing connected components with no more than five pixels. From Fig. 6, our hierarchical models have generally better results than the corresponding fine-level methods since non-stationary backgrounds can be well pre-processed in coarse level.

To evaluate our method, a measurement presented by Li et al. [4] is used in this paper. Let $A$ be a detected region and $B$ be the corresponding ground truth, the similarity between $A$ and $B$ is defined as

$$
S(A, B)=\frac{A \cap B}{A \cup B} .
$$

$S(A, B)$ lies between 0 and 1 . If $A$ and $B$ are the same, $S(A, B)$ approaches 1 , otherwise 0 if $A$ and $B$ have the least similarity. The ground truths are marked manually. The evaluation results are shown in Table 3, which reveals that the proposed hierarchical approach can provide an averagely 
Table 2

Coarse-level processing speeds in fps. Five benchmark sequences shown in Table 1 are used for evaluation

\begin{tabular}{lll}
\hline Sequence name & $\begin{array}{l}\text { Our coarse-level } \\
\text { method }\end{array}$ & $\begin{array}{l}\text { LBP-based } \\
\text { method }\end{array}$ \\
\hline Fountains & 67.12 & 37.66 \\
Waving leaves & 68.43 & 36.79 \\
Ocean waves & 68.22 & 37.64 \\
Waving river & 69.77 & 39.49 \\
Moving escalator & 69.63 & 43.35 \\
Average & 68.63 & 38.99 \\
\hline
\end{tabular}

Table 3

Quantitative evaluations by using the $S(A, B)$ measure

\begin{tabular}{lll}
\hline Sequence name & $\begin{array}{l}\text { Hierarchical method } \\
\text { (using MoG) }\end{array}$ & MoG \\
\hline Fountains & 0.6422 & 0.4868 \\
Waving leaves & 0.6084 & 0.5037 \\
Ocean waves & 0.4840 & 0.4463 \\
Waving river & 0.5353 & 0.1558 \\
Moving escalator & 0.0771 & 0.0628 \\
Average & 0.4694 & 0.3311 \\
\hline
\end{tabular}

Table 4

Fine-level processing speeds in fps and five benchmark sequences shown in Table 1 are used for evaluation

\begin{tabular}{llc}
\hline Algorithm & $\begin{array}{l}\text { Hierarchical method } \\
\text { (using MoG) }\end{array}$ & MoG \\
\hline Fountains & 22.03 & 29.22 \\
Waving leaves & 21.09 & 27.61 \\
Ocean waves & 21.59 & 24.98 \\
Waving river & 19.43 & 25.12 \\
Moving escalator & 21.81 & 28.50 \\
Average & 21.19 & 27.09 \\
\hline
\end{tabular}

better performance than those of its fine-level methods adopted.

Results of processing speed are shown in Tables 2 and 4. The average speed of the proposed color-version coarse-level method is $68.63 \mathrm{fps}$, and that of the hierarchical method is $21.19 \mathrm{fps}$ when MoG is adopted in fine level. The average speed of MoG is $27.09 \mathrm{fps}$. For MoG, the sum of the processing times of running the coarse- and fine-level methods independently is $0.0515(=1 / 68.63+1 / 27.09) \mathrm{s}$. This result in $19.42 \mathrm{fps}$ that is slower than $21.19 \mathrm{fps}$ achieved by the hierarchical method when MoG is adopted in fine level. The experiments demonstrated that our method is more efficient than that of just intersecting the coarse and fine results.

\section{Conclusion and future work}

A two-level scalable detector is presented in this paper. We propose an effective block-based descriptor, contrast histogram, for coarse background model. We also propose an asymmetric feed-forward strategy to efficiently combine coarse and fine background models. The advantage with this hierarchical structure is that non-stationary background can be identified, and rough foreground objects can be detected in coarse level in advance. Then based on the result of coarse block level, fine pixel-level method is performed to further extract the detailed shapes of foreground objects. Our approach is practically useful since it can be realized very fast, and both coarse and fine segmentations are affordable in a single framework.

As to our knowledge, this is the first approach providing scalability in background-modeling-based foreground detection. Our method allows a very efficient coarse foreground to be available at first, and then a fine foreground latter. The scalable property is suitable for applications that need variable rates/qualities of detections, such as in IP camera or intelligent camera. Quantitative evaluations and comparative studies show that the proposed hierarchical method can further improve the performances of the adopted fine-level methods by incorporating the coarse-level information as priori knowledge.

Currently, the integrated processing speed of coarse and fine levels is faster than executing them in turn independently. In the future, we plan to further speed up the integration so that the integrated speed is comparable to that of executing the finelevel method only.

\section{Acknowledgments}

The authors would like to thank the helpful comments and suggestions given by the reviewers. This work was supported in part under Grants 95-EC-17-A-02-S1-032 and NSC 95-2752E-002-007-PAE.

\section{References}

[1] A. Elgammal, D. Harwood, L.S. Davis, Non-parametric model for background subtraction, in: Proceedings of European Conference on Computer Vision, 2000, pp. 751-767.

[2] T. Horprasert, D. Harwood, L.S. Davis, A statistical approach for real-time robust background subtraction and shadow detection, in: Proceedings of IEEE ICCV Frame-Rate Workshop, 1999, pp. 1-19.

[3] N. Martel-Brisson, A. Zaccarim, Moving cast shadow detection from a gaussian mixture shadow model, in: Proceedings of IEEE Conference on Computer Vision and Pattern Recognition, 2005, pp. 643-648.

[4] L. Li, W. Huang, I.Y.H. Gu, Q. Tian, Statistical modeling of complex backgrounds for foreground object detection, IEEE Trans. Image Process. 13 (11) (2004) 1459-1472.

[5] K. Toyama, J. Krumm, B. Brumitt, B. Meyers, Wallflower: principles and practice of background maintenance, in: Proceedings of International Conference on Computer Vision, 1999, pp. 255-261.

[6] C.R. Wren, A. Azarbayejani, T. Darrell, A.P. Pentland, Pfinder: real-time tracking of the human body, IEEE Trans. Pattern Anal. Mach. Intell. 19 (7) (1997) 780-785.

[7] C. Stauffer, W.E.L. Grimson, Adaptive background mixture models for real-time tracking, in: Proceedings of IEEE Conference on Computer Vision and Pattern Recognition, 1999, pp. 246-252. 
[8] M. Harville, G. Gordon, J. Woodfill, Foreground segmentation using adaptive mixture models in color and depth, in: Proceedings of IEEE Workshop on Detection and Recognition of Events in Video, 2001, pp. $3-11$.

[9] M. Harville, A framework for high-level feedback to adaptive, per-pixel, mixture-of-gaussian background models, in: Proceedings of European Conference on Computer Vision, 2002, pp. 543-560.

[10] D.S. Lee, Effective gaussian mixture learning for video background subtraction, IEEE Trans. Pattern Anal. Mach. Intell. 27 (5) (2005) 827-832.

[11] C. Ridder, O. Munkelt, H. Kirchner, Adaptive background estimation and foreground detection using kalman-filtering, in: Proceedings of International Conference on Recent Advances in Mechatronics, 1995, pp. 193-199.

[12] J. Zhong, S. Sclaroff, Segmenting foreground objects from a dynamic textured background via a robust kalman filter, in: Proceedings of International Conference on Computer Vision, 2003, pp. 44-50.

[13] B. Stenger, V. Ramesh, N. Paragios, F. Coetzee, J.M. Buhmann, Topology free hidden Markov models: application to background modeling, in: Proceedings of IEEE International Conference on Computer Vision, 2001, pp. 294-301.

[14] K. Kim, T.H. Chalidabhongse, D. Harwood, L.S. Davis, Real-time foreground-background segmentation using codebook model, Real-Time Imaging 11 (3) (2005) 167-256.
[15] T. Matsuyama, T. Ohya, H. Habe, Background subtraction for nonstationary scenes, in: Proceedings of Asian Conference on Computer Vision, 2000, pp. 662-667.

[16] M. Mason, Z. Duric, Using histograms to detect and track objects in color video, in: Proceedings of 30th Applied Imagery Pattern Recognition Workshop, 2001, pp. 154-159.

[17] A. Monnet, A. Mittal, N. Paragios, V. Ramesh, Background modeling and subtraction of dynamic scenes, in: Proceedings of IEEE International Conference on Computer Vision, 2003, pp. 1305-1312.

[18] M. Heikkilä, M. Pietikäinen, J. Heikkilä, A texture-based method for detecting moving objects, in: Proceedings of British Machine Vision Conference, 2004, pp. 187-196.

[19] T. Ojala, M. Pietikainen, T. Maenpaa, Multiresolution gray-scale and rotation invariant texture classification with local binary patterns, IEEE Trans. Pattern Anal. Mach. Intell. 24 (7) (2002) 971-987.

[20] C.R. Huang, C.S. Chen, P.C. Chung, Contrast context histogram-A discriminating local descriptor for image matching, in: Proceedings of IEEE International Conference on Pattern Recognition, 2006, pp. $53-56$.

[21] D.G. Lowe, Distinctive image features from scale-invariant keypoints, Int. J. Comput. Vision 60 (2) (2004) 91-110.

[22] R.C. Gonzalez, R.E. Woods, Digital Image Processing, Section 2.5.2, Prentice-Hall, Englewood Cliffs, NJ, 2002.

\begin{abstract}
About the Author-YU-TING CHEN received the B.S. and M.S. degrees in the Department of Graphic Arts Communication from National Taiwan Normal University, Taipei, Taiwan, in 2000 and 2003, respectively. He is currently a research assistant at the Institute of Information Science, Academia Sinica, Taipei, Taiwan. He is also pursuing a doctorate degree in computer science and information engineering at National Taiwan University, Taipei, Taiwan. His research interests include image processing, computer vision, surveillance, and pattern recognition.
\end{abstract}

About the Author-CHU-SONG CHEN received a B.S. in Control Engineering from National Chiao-Tung University, Hsing-Chu, Taiwan, in 1989. He received an M.S. in 1991 and a Ph.D in 1996, respectively, both from the Department of Computer Science and Information Engineering, National Taiwan University. $\mathrm{He}$ is now an associate research fellow of the Institute of Information Science, Academia Sinica, and an adjunct associate professor of Graduate Institute of Networking and Multimedia, National Taiwan University. His research interests include computer vision, pattern recognition, and image/video analysis.

About the Author-CHUN-RONG HUANG received the B.S. and Ph.D. degrees in the Department of Electrical Engineering from National Cheng Kung University, Taiwan, in 1999 and 2005, respectively. In 2005, he joined the Institute of Information Science, Academia Sinica, and has become a Post-doctoral Fellow since 2005. Dr. Huang's research interests include computer vision, computer graphics, pattern recognition, image processing, and medical image processing.

About the Author-YI-PING HUNG received his B.Sc. in Electrical Engineering from the National Taiwan University in 1982. He received an M.Sc. from the Division of Engineering, an M.Sc. from the Division of Applied Mathematics, and a Ph.D. from the Division of Engineering, all at Brown University, in 1987, 1988, and 1990, respectively. He is currently a professor in the Graduate Institute of Networking and Multimedia, and in the Department of Computer Science and Information Engineering, both at the National Taiwan University. From 1990 to 2002, he was with the Institute of Information Science, Academia Sinica, Taiwan, where he became a tenured research fellow in 1997 and is now an adjunct research fellow. He received the Young Researcher Publication Award from Academia Sinica in 1997. His current research interests include computer vision, pattern recognition, image processing, virtual reality, multimedia, and human-computer interaction. 